\title{
Comparación de cianuro y tiourea como agentes lixiviantes de un mineral aurífero colombiano
}

\author{
Comparison Between Cyanide and Thiourea, as Leaching Agents \\ of a Colombian Gold Ore
}

Fecha de Recepción: 2013-06-10

Fecha de Aprobación: 2013-06-29

\section{Resumen}

Estudio preliminar de la lixiviación del oro a partir de un mineral aurífero procedente de Marmato (Caldas); como agentes lixiviantes se emplearon tiourea y cianuro sódico. El estudio se desarrolló en tres etapas: 1) caracterización del mineral mediante Difracción de Rayos $\mathrm{X}$ (DRX), Ensayo al Fuego y cuantificación de oro por Absorción Atómica (AA); 2) realización de lixiviaciones con los agentes a muestras minerales de $100 \mathrm{~g}$, durante 8 horas, con una agitación de $800 \mathrm{rpm}$ y temperatura ambiente, tomando muestras de solución a intervalos controlados de tiempo, y 3 ) análisis
José Isaí Santamaría Roncancio* Robinson de Jesús Torres Gómez** Mario Parra Pinilla***

César Armando Ortiz Otálora****

\section{Abstract}

A preliminary study was made by leaching gold from a gold ore from Marmato (Departament of Caldas). As leaching agents were used thiourea and sodic cyanide. The study was divided into three stages: The first one, was the ore characterization by X-ray diffraction (XRD), plus gold fire essay and a quantification by Atomic Absorption (AA). In the second stage, leaching was carried out, on mineral samples agents of $100 \mathrm{~g}$ during 8 hours, with agitation at $800 \mathrm{rpm}$ and environmental temperature, taking solution samples at controlled time intervals. Third, an analysis of the gold

\footnotetext{
* Universidad Pedagógica y Tecnólogica de Colombia (Colombia).jose.santameria@uptc.edu.co

** M.Sc. Universidad Pedagógica y Tecnólogica de Colombia (Colombia).robinson.torres@uptc.edu.co

*** M.Sc. Universidad Pedagógica y Tecnólogica de Colombia (Colombia).mario.parra@uptc.edu.co

${ }^{\star * \star *}$ M.Sc. Universidad Pedagógica y Tecnólogica de Colombia (Colombia).cesar.ortiz@uptc.edu.co
} 
de rendimientos de extracción de oro. Las lixiviaciones con tiourea arrojaron mayor rendimiento que las de cianuro.

Palabras clave: Lixiviación, Agentes lixiviantes, Tiourea, Cianuro, Oro. extraction yields. The thiourea leaching tests out performed the cyanide ones.

Keywords: Leaching, Leaching Agents, Thiourea, Cyanide, Gold. 


\section{INTRODUCCIÓN}

Es misión de la metalurgia extractiva separar los metales de mayor interés, hasta producirlos en un estado de relativa pureza para poderlos utilizar. La industria minera, en particular la asociada a la explotación de los metales preciosos, indiscutiblemente ha tenido un peso importante en el desarrollo de la economía mundial a lo largo de la historia. Desde antes de la llegada de los españoles, en Colombia se han explotado minas que contienen metales preciosos, y más recientemente se han buscado mejoras en los procesos de extracción y se han impuesto regulaciones mineras que tratan de limitar el uso de ciertos agentes. El proceso de lixiviación con cianuro se ha empleado con "éxito" desde finales del siglo XIX en la obtención de oro y plata, pero debido a los efectos negativos sobre el medioambiente de este agente y a las subsecuentes restricciones establecidas, se buscan nuevas alternativas, que generen excelentes porcentajes de extracción y una mejor aceptación ante las entidades mineras de control ambiental; por esta razón, el presente estudio busca implementar la tiourea, haciendo lixiviaciones de oro con ambos métodos, para comparar el rendimiento en la recuperación de oro y generar procesos alternativos en la industria minera nacional.

\section{Procedimiento Experimental}

\section{A. Pruebas y materiales}

\section{1) Cuarteado y preparación mecánica del} mineral: El cuarteo es una operación posterior al muestreo, la cual nos permite continuar disminuyendo la cantidad de muestra hasta tener una muestra "promedio" y relativamente representativa de un todo de gran volumen. Generalmente, el cuarteo realizado en el laboratorio es el de por conos [1].
2) Difracción de rayos $X(D R X)$ : La difracción de rayos $X$ es uno de los fenómenos físicos que se producen al interaccionar un haz de rayos $\mathrm{X}$, de una determinada longitud de onda, con una sustancia cristalina [2]. Esta técnica permite determinar las fases cristalinas presentes en el mineral aurífero; en este proceso se utilizó el equipo de difracción de rayos X Panalitical xpert-pro, con un ánodo de $\mathrm{Cu}$ K-Alphal $[\AA]$ : 1,54060 , configuración de generador de $40 \mathrm{~mA}, 45 \mathrm{kV}$ y con un barrido de 20 a 110 grados, tamaño de paso de 0,02 grados y tiempo de conteo de 4 segundos.

3) Ensayo alfuego: El conocido procedimiento de ensayo al fuego genera resultados cuantitativos de metal de interés en un mineral. Las muestras se mezclan con agentes fundentes que incluyen el óxido de plomo (litargirio) y se funden a alta temperatura. El óxido de plomo se reduce a plomo, que colecta el metal precioso. Cuando la mezcla fundida solidifica, el plomo permanece en el fondo [3].

Fusión del mineral: Del mineral pulverizado se tomaron $80 \mathrm{~g}$, que fueron divididos en cuatro partes iguales y mezclados con el material fundente dentro de los crisoles, que se llevaron a un horno (mufla) a una temperatura de 1120 ${ }^{\circ} \mathrm{C}$, hasta que el material sólido se fundió. Las coquillas de acero fueron precalentadas para hacer el proceso de colada con un leve movimiento, para asegurar que todo el plomo se precipite junto con el oro y la plata; una vez solidificado el material se le desprendió toda la escoria, formando un cubo en caliente con el $\mathrm{Pb}, \mathrm{Au}-\mathrm{Ag}$ para proceder a la copelación.

Copelación: Los cubos obtenidos del proceso de fusión se depositaron en copelas y se llevaron a un horno tipo mufla a temperatura de $950^{\circ} \mathrm{C}$, donde la mayoría del $\mathrm{PbO}$ es absorbido por la 
copela, y el resto se volatiliza, quedando como residuo un dore brillante formado por Ag-Au.

Refinación del dore: El dore fue pesado y luego laminado, para pasar a la separación de la plata en una solución en caliente (105 ${ }^{\circ} \mathrm{C}$ ) de agua y ácido nítrico en proporción 1:5, respectivamente; para darle brillo al oro, por la tonalidad opaca, se lava la lámina en ácido nítrico puro; de nuevo la lámina es pesada y por diferencia de pesos es encontrada la cantidad de oro y plata presentes en el mineral.

4) Espectroscopia de absorción atómica: $\mathrm{La}$ espectroscopia de absorción atómica se emplea en la determinación cualitativa y cuantitativa de aproximadamente 70 elementos. La sensibilidad de los métodos atómicos está en los límites de partes por millón a partes por mil millones [5-6].

El proceso de absorción atómica para la calibración del equipo se hizo con una flama de acetileno/aire; se instaló la lámpara de oro a $12 \mathrm{~mA}$ de corriente y $324,8 \mathrm{~nm}$ de longitud de onda. Se prende la flama y se le da el tamaño aproximado de $10 \mathrm{~cm}$ de alto y color azul intenso, haciendo pasar por el capilar cada muestra patrón y las muestra de lixiviación tomando la lectura emitida de absorción [6]

Digestión del mineral. Del mineral pulverizado se sacaron $60 \mathrm{~g}$, divididos en tres partes iguales y vertidos en un balón aforado de base plana en solución con agua regia; esta mezcla se puso sobre una estufa de agitación a $800 \mathrm{rpm}$ durante seis horas, logrando de esta forma la disolución del oro metálico presente en el mineral.

5) Cianuración: En esta etapa se efectuaron tres lixiviaciones con 100 g para cada una de las siguientes concentraciones de cianuro: 0,011,
0,012 y $0,013 \mathrm{~g} / 0.150 \mathrm{~L}$, respectivamente. El proceso de lixiviación se hizo con agitación mecánica a $800 \mathrm{rpm}$ durante ocho horas, $\mathrm{pH}$ 10.5 a temperatura y presión ambiente.

6) Tioureación: Se tomaron nueve muestras de 100 g cada una; para este proceso de tioureación se empleó $\mathrm{Fe}^{+3}$, ácido sulfúrico, tiourea y $\mathrm{pH}$ comprendido entre 1.5-2.5, con agitación mecánica de $800 \mathrm{rpm}$, durante un periodo de ocho horas.

\section{Análisis de Resultados}

\section{A. Caracterización del mineral}

1) Difracción de rayos $X$ (DRX): Luego del ensayo pudo establecerse que se trata de un mineral complejo, que no se puede clasificar como silícico o ferruginoso. Se hace mención especial a las fases que contienen hierro y cobre, porque son las que pueden elevar el consumo de agente lixiviante. Además, conociendo las especies minerales presentes se diseñó la mezcla adecuada para efectuar los ensayos al fuego.

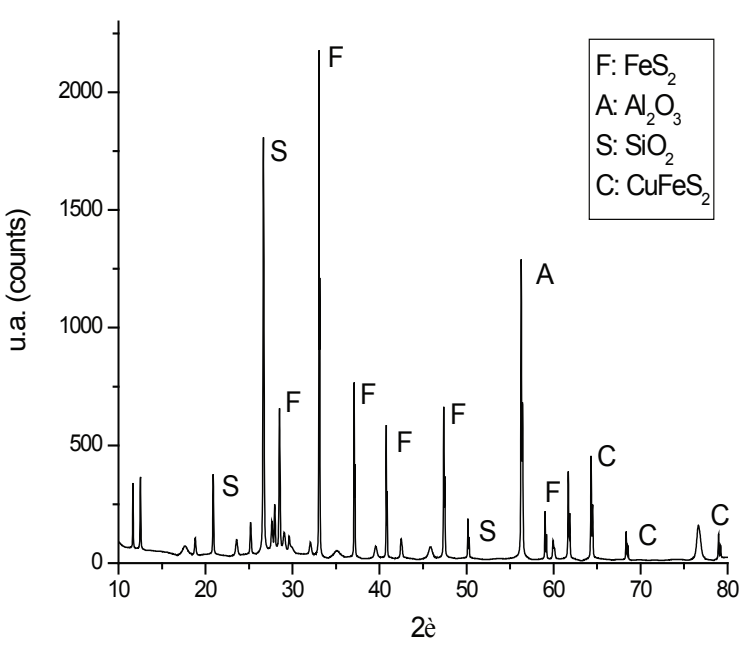

Gráfica 1. Difractograma de rayos X con las especies presentes en el mineral de estudio. 
2) Ensayo al fuego: Luego de la copelación, el dore fue pesado, encontrándose que cada tonelada de mineral de estudio contenía 14.766 g de Au-Ag. Luego de hacer la separación de oro y plata se encontró una pérdida en peso de $0.000003 \mathrm{~g}$, que pertenecen a la cantidad de plata contenida en el dore. Los $0.000292 \mathrm{~g}$ pertenecen a la cantidad de oro presentes en 20 $\mathrm{g}$ de mineral; por consiguiente, tendremos que por cada tonelada de mineral aurífero existen $14.60 \mathrm{~g}$ de oro.

3) Espectroscopia de absorción atómica: De la solución madre de oro, de 1000 ppm, se tomaron alicuotas para preparar tres soluciones (patrón) diluidas de 11 ppm, 15 ppm y 27 ppm.

El equipo de espectrometría de absorción atómica fue calibrado según el procedimiento para el Perkin Elmer 3110. De esta forma se encuentra que cada tonelada de mineral tendrá $14.27 \mathrm{~g}$ de $\mathrm{Au}$; como ya se calculó la cantidad de oro contenido en el mineral por análisis de ensayo al fuego, se promediaron estos dos resultados, originando un resultado final de $14.43 \mathrm{~g}$ de oro por tonelada de mineral.

\section{B. Procesos de lixiviación}

1) Lixiviación con cianuro. En la gráfica 2 , que describen el proceso de cianuración; el comportamiento de las curvas tiene tres áreas o zonas definidas que describen la cantidad de oro disuelto en la solución y la actividad de reacción o agresividad del cianuro en el mineral. El área 1 tiene porcentajes de bajo nivel, es decir, el cianuro no ha reaccionado -etapa de duración aproximada de tres horas y media-; en las siguientes dos horas se presenta el área 2, donde el cianuro ha subido su agresividad con el porcentaje de disolución de oro; además de gastarse el cianuro en el oro, los agentes cianicidas siguen avanzando en el consumo de agente lixiviante. A medida que aumenta la concentración de agente lixiviante aumenta el porcentaje de metal de interés extraído. Al valorar la curva de mayor concentración de cianuro se tiene el mejor rendimiento en el proceso de lixiviación de mineral aurífero con este agente en un periodo de ocho horas continuas de trabajo. Es posible alcanzar mayores porcentajes en rendimiento sabiendo que la reactividad del cianuro puede tardar de 16 a 48 horas [7].

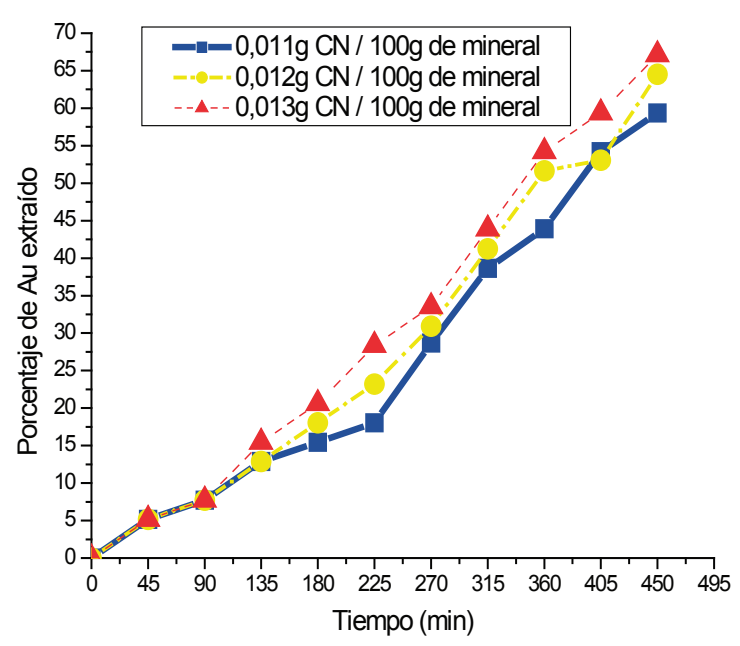

Gráfica 2. Extracción de oro comparativa con las tres concentraciones de cianuro

2) Lixiviación con tiourea: Para las tioureaciones se utilizaron tres dosificaciones distintas de agente lixiviante. Las lixiviaciones con tiourea ofrecen gran reactividad en tiempos comprendidos de 8 a 16 horas [8], razón por la cual las curvas de la gráfica de este proceso ofrecen información detallada del aumento de extracción de oro con respecto al tiempo y control de $\mathrm{pH}$; a medida que transcurre el tiempo, la reactividad de la tiourea es mayor, y manteniendo el $\mathrm{pH}$ entre 1.8 y 2.5 , rango de mayor activación $\mathrm{y}$, por consiguiente, mejor recuperación de metal de interés.

Cuando se trabajó concentración de $1.5 \mathrm{~g}$ de agente lixiviante se logró el mayor rendimiento con respecto a las demás concentraciones, como lo muestra la gráfica 3 . 


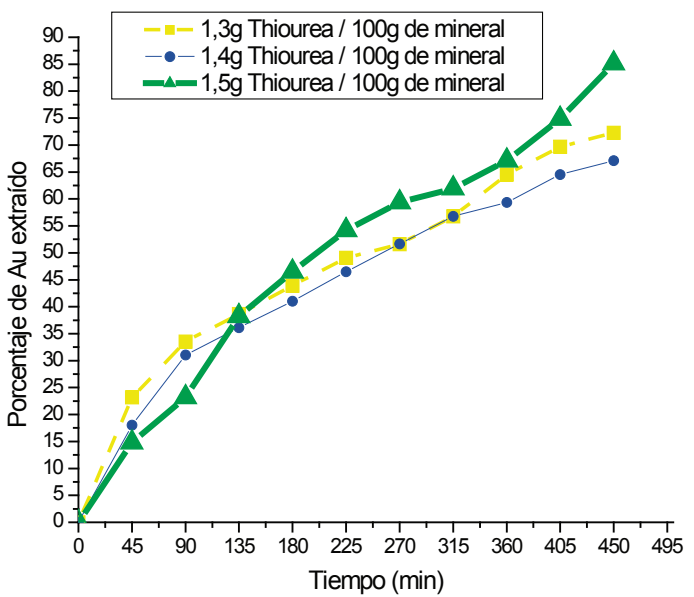

Gráfica 3. Extracción de oro comparativa con las tres concentraciones de tiourea

\section{Comparación de los procesos de lixiviación}

Para elaborar la gráfica 4 se tomó el mejor resultado porcentual de dilución de oro en los licores de cada proceso de lixiviación. Se puede concluir con cada curva cuál agente ataca con mayor eficiencia al oro encapsulado dentro del mineral. Los procesos de lixiviación se hicieron con las mismas condiciones de trabajo, se observa que el comportamiento de la tiourea da mejores resultados en menores tiempos de trabajo en la recuperación de oro.

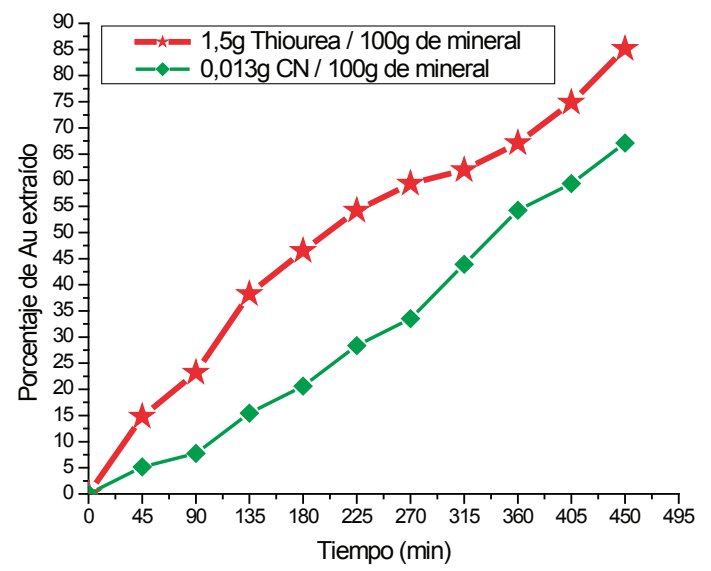

Gráfica 4. Comparación de los mejores resultados en los procesos de lixiviación

\section{Conclusiones}

Los procesos de identificación de las especies químicas presentes en el mineral generan información básica para realizar ensayos al fuego, de tal forma que se pueda caracterizar la mayor cantidad de oro y escoger el modelo conveniente de extracción de metal.

La dosificación óptima de cianuro corresponde a $0,013 \mathrm{~g}$ por cada $100 \mathrm{~g}$ de mineral.

La mejor extracción de oro desde el mineral se obtuvo cuando se agregó $1,5 \mathrm{~g}$ de tiourea por cada $100 \mathrm{~g}$ de mineral.

Las lixiviaciones con cianuro en ocho horas de trabajo dan rendimientos de recuperación de oro del mineral del orden de $68.78 \%$, y con tiourea, $87.27 \%$; esta ganancia se da porque la reacción de la tiourea se logra en menor tiempo que el cianuro; por esta razón se puede decir que la tiourea, en el desarrollo de este estudio, es mejor agente lixiviante.

En el proceso de cianuración se trabajó con $\mathrm{pH}$ de 9.5 a 10.6 , y en la tioureación con $\mathrm{pH}$ de 1.8 a 2.7 ; controlado el $\mathrm{pH}$ dentro de estos rangos se logra mayor recuperación de oro.

Se puede concluir que la utilización de la tiourea para extraer oro desde un mineral es una tecnología novedosa y sencilla, comparada con otros agentes lixiviantes, pudiendo competir con la cianuración.

\section{REFERENCIAS}

[1] N. Linares, Procesamiento de minerales con oro y plata, Perú, 2008.

[2] P. J. Bermúdez, Teoría y práctica de la espectroscopia de Rayos X, España:Alhambra, 1967. 
[3] I. Reyes, Metalurgia del oro y medioambiente-Unt-Diciembre, 1997.

[4] D. A. Skoog, J. J. Leary, Análisis instrumental. Cuarta edición, España: McGraw-Hill, 1995.

[5] D. A. Skoog, D. M. West, J. F. HOLLER, Análisis instrumental, sexta edición, España: McGraw-Hill, 1999.

[6] Perkin Elmer Company, Model 3110 Atom absorption spectrometer users guide.
[7] N. Gönen, "Leaching of finely disseminated gold ore with cyanide and thiourea solutions". Hidrometalurgia, 69, 2003, p. 169-176.

[8] M. E. Poisot-Díaz, "Electro deposition of a Silver-Gold Alloy (DORÉ) from Thiourea Solutions in the Presence of Other Metallic Ion Impurities". Hydrometallurgy, 93, 2007, p. 21-27. 